\section{Evaluation of optic disc cupping using high-resolution ocular ultrasound}

\author{
Abstract \\ Background/Purpose The new Biovision \\ B-Scan Probe has greater resolution than its \\ predecessors because of its posteriorly placed \\ focus (25 $\mathrm{mm}$ inside the eye) and the \\ consequent reduction in size of the sound \\ field. We investigated the accuracy, \\ reproducibility and test-retest variability of \\ two-dimensional optic cup measurements \\ using this new-generation B-scanner. \\ Methods One randomly selected eye of 20 \\ patients underwent five repeated \\ measurements of vertical (VOCD) and \\ horizontal optic cup diameters (HOCD), and \\ optic cup depth, using confocal scanning laser \\ tomography (Heidelberg Retinal Tomograph) \\ and high-resolution ultrasound (Biovision, \\ Chiron Vision, UK) on two separate occasions. \\ There was one operator per instrument who \\ was masked to all clinical data and previous \\ measurements. Accuracy of ultrasound \\ readings was quantified by comparing the \\ results with those of Heidelberg Retinal \\ Tomography (HRT). \\ Results The $95 \%$ confidence interval for the \\ bias of echographic vertical and horizontal cup \\ diameter measurements was $-95 \pm 48 \mu \mathrm{m}$ and \\ $-19 \pm 72 \mu \mathrm{m}$ respectively, and $-87 \pm 328 \mu \mathrm{m}$ \\ for cup depth readings. The indices of \\ reproducibility (mean coefficient of \\ repeatability \pm SD) were: VOCD: $177 \pm 105$ \\ $\mu \mathrm{m}$ [B-scan], $209 \pm 100 \mu \mathrm{m}$ [HRT]; HOCD: \\ $179 \pm 61 \mu \mathrm{m}$ [B-scan], $205 \pm 101 \mu \mathrm{m}$ [HRT]; \\ cup depth: $206 \pm 63 \mu \mathrm{m}$ [B-scan], $204 \pm 124 \mu \mathrm{m}$ \\ [HRT]. Ninety-five per cent limits of \\ agreements between initial and retest values \\ for ultrasound were $18 \pm 136 \mu \mathrm{m}$ [VOCD], \\ $2 \pm 144 \mu \mathrm{m}$ [HOCD] and $4 \pm 156 \mu \mathrm{m}$ [cup \\ depth], compared with $1 \pm 104 \mu \mathrm{m}$ [VOCD], \\ $20 \pm 102 \mu \mathrm{m}$ [HOCD] and $3 \pm 168 \mu \mathrm{m}$ [cup \\ depth] for scanning laser tomography. \\ Conclusion The results demonstrate that \\ measurements of optic cup diameter and \\ depth using ultrasound correlate strongly with \\ corresponding HRT readings. These \\ echographic measurements are reproducible, \\ and not subject to clinically meaningful \\ test-retest variability. This technique of \\ measuring two-dimensional cup parameters
}

\author{
S. BEATTY, P.A. GOOD, \\ J. MCLAUGHLIN, M. TSALOUMAS, \\ E.C. O'NEILL
}

does not require expensive specialist equipment and has many potential clinical applications which are discussed.

Key words Glaucoma, Optic cup, Retinal tomography, Ultrasound

Assessment of the glaucoma patient includes tonometry, perimetry and morphological evaluation of the optic disc. The reliability and accuracy of laser tomographic scanning devices in the morphometric analysis of the optic nerve head have been established. ${ }^{1-3}$ However, this method involves expensive specialist equipment and image quality may be affected by media opacities. The present study was conducted to determine whether echographic measurements of the optic cup using a new generation B-scanner could be used in the diagnosis and follow-up of glaucoma patients.

\section{Materials and methods}

Twenty patients attending BMEC for visual field testing were recruited into the study. Of these, 16 suffered from primary open angle glaucoma and the remainder had a diagnosis of ocular hypertension. Subjects were informed of the nature of the study and consent was obtained. The only inclusion criterion was clear optic media.

One randomly selected eye of each subject underwent optic disc morphometry (performed with the Heidelberg Retinal Tomograph, Heidelberg [Germany] Engineering) and measurements of two-dimensional parameters of the optic cup (performed with Biovision BScan ultrasound) on two separate occasions. For all measurements, five repeated readings were taken and the mean calculated. There was one operator only for each instrument (S.B., Heidelberg Retinal Tomography; P.G., ultrasound), and Heidelberg Retinal Tomography was performed before ultrasound in all cases. Both operators were masked to prior measurements and clinical information. 


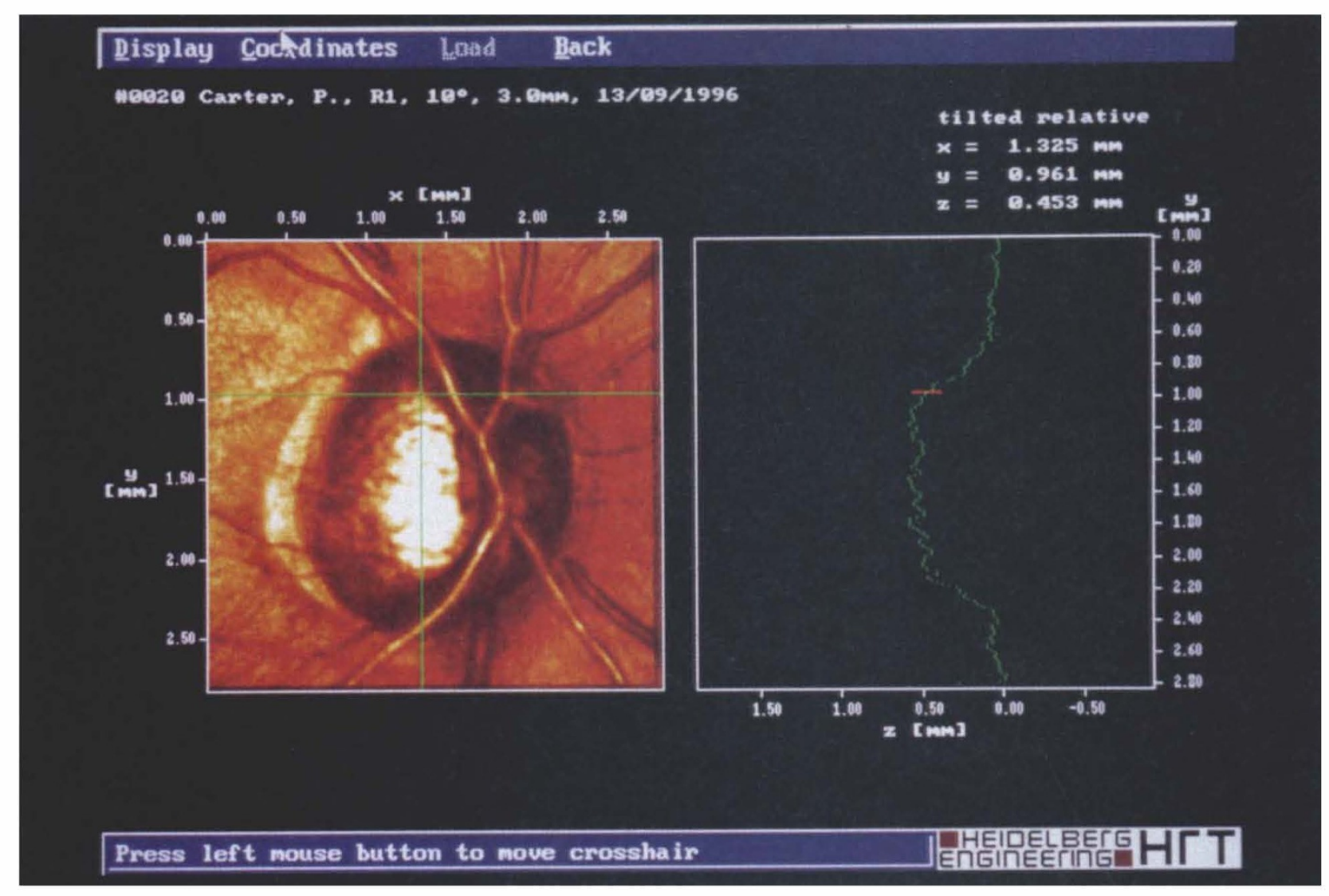

Fig. 1. Topographic image (right) and Y-profile (left) of the optic nerve head produced by Heidelberg Retinal Tomography (HRT).

\section{Instrumentation}

Scanning laser tomography

Heidelberg Retinal Tomography (HRT) images the retina in three dimensions and therefore provides topographic measurements. This is achieved by the projection of a diode laser beam $(670 \mathrm{~nm})$ via a confocal system. The confocal system ensures that only light reflected from a defined focal plane, which the HRT places on the retina, is detected by the integrated photomultiplier. This main focal plane is a reference plane to which all elevations and depressions of the fundus are related.

To obtain three-dimensional measurements, HRT acquires 32 equally spaced confocal images along the $z$-axis (perpendicular to the optical axis). The image resolution is $256 \times 256$ pixels, resulting in 65536 measurements per image. The operator defines the depth of the scanning range, which can be raised from $0.5 \mathrm{~mm}$ to $4 \mathrm{~mm}$. The scanned field size can also be raised $\left(10^{\circ} \times 10^{\circ}, 15^{\circ} \times 15^{\circ}\right.$ or $\left.20^{\circ} \times 20^{\circ}\right)$. Reflectivity along the $z$-axis is used to generate a topographic image, measured in micrometres from the main focal plane.

To allow three-dimensional measurements of the disc, the margin of the optic nerve head has to be defined by the operator. The height values of the contour line are corrected for the influence of crossing vessels by an automatic interpolation. The calculation of the morphometric parameters is not influenced by the operator (i.e. HRT automatically computes these data for the entire nerve head on advice).
We obtained 10 independent $10^{\circ} \times 10^{\circ}$ images per eye, centred on the optic nerve, during two separate sessions (five images per session). The pupil was not dilated in any case. After each acquisition, the instrument verified that exposure was correct; images with improper exposure or eye movement were excluded.

The images were stored in a file on the hard disk drive of the instrument and the following parameters computed: disc area; cup area; cup/disc area ratio; rim area; cup volume; rim volume; mean cup depth; maximum cup depth; mean retinal nerve fibre layer (RNFL) thickness and RNFL cross-section area.

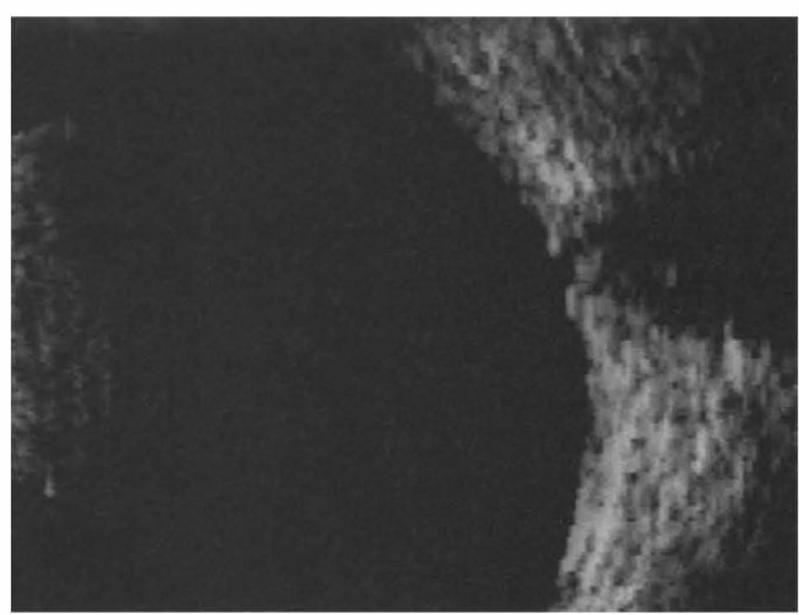

Fig. 2. Axial B-scan display showing the optic cup as a posterior concavity anterior to the acoustically clear area of the optic nerve. 
Table 1. Estimates of two-dimensional optic cup parameters, and the indices of reproducibility and test-retest variability, as measured by echography and scanning laser tomography

\begin{tabular}{|c|c|c|c|c|c|c|}
\hline & \multicolumn{2}{|c|}{$\begin{array}{l}\text { Mean } \pm S D \\
(\mu \mathrm{m})\end{array}$} & \multicolumn{2}{|c|}{$\begin{array}{l}\text { Coefficient of repeatability } \\
\pm \mathrm{SD}(\mu \mathrm{m})\end{array}$} & \multicolumn{2}{|c|}{$\begin{array}{l}\text { Test-retest variability }(\mu \mathrm{m}) \\
\text { (mean of differences } \pm 2 \mathrm{SD}\end{array}$} \\
\hline & HRT & $\mathrm{U} / \mathrm{S}$ & HRT & $\mathrm{U} / \mathrm{S}$ & HRT & $\mathrm{U} / \mathrm{S}$ \\
\hline Maximum vertical optic cup diameter & $933 \pm 296$ & $838 \pm 295$ & $177 \pm 105$ & $209 \pm 100$ & $1 \pm 104$ & $18 \pm 136$ \\
\hline Maximum horizontal optic cup diameter & $837 \pm 245$ & $818 \pm 305$ & $205 \pm 101$ & $179 \pm 61$ & $20 \pm 102$ & $2 \pm 144$ \\
\hline Maximum optic cup depth & $711 \pm 245$ & $624 \pm 245$ & $204 \pm 124$ & $206 \pm 63$ & $3 \pm 168$ & $4 \pm 156$ \\
\hline
\end{tabular}

HRT, Heidelberg Retinal Tomography; U/S, ultrasound.

The maximum vertical optic cup diameter was calculated as follows. First, the topographic image of the optic nerve head was displayed on the monitor. Next, under guidance of the $Y$-profile (Fig. 1), the maximum VOCD (the greatest vertical distance 'below contour') was defined. Finally, the distance between markers placed at each end of the maximum vertical optic cup diameter was calculated by the computer. The maximum horizontal optic cup diameter was calculated in the same way, but using the $X$-profile as a guide.

\section{Ultrasound}

The poor resolution of the posterior pole traditionally associated with ultrasound reflects the considerable widening of the sound field that occurs after reflection from a concave spherical surface. This increase in size of the field occurs because the focal point for the reflected ultrasound lies at a distance of one-half the radius of curvature in front of the posterior pole. We used a newgeneration B-Scanner (Biovision B-Scan) which is focused at $27 \mathrm{~mm}$ from the concave transducer (i.e. $25 \mathrm{~mm}$ inside the eye), allowing greater image resolution of structures at the posterior pole.

Using this instrument the following two-dimensional parameters of the optic cup were measured: maximum vertical optic cup diameter; maximal horizontal optic cup diameter; maximum optic cup depth. The ultrasound probe is orientated in the appropriate plane until the desired view of the optic cup is obtained. This image is then frozen and displayed on the monitor (Fig. 2).

Maximum vertical optic cup diameter was defined as the greatest distance between the superior and inferior lips of the posterior concavity of the optic nerve head, whereas maximum horizontal optic cup diameter was estimated as the greatest distance between the right and left lips of the cup. Maximum cup depth was judged to be the greatest distance between the base of the cup and a point midway between the edges of the cup. All distances were computed by the instrument. For each measurement, five readings were taken and the mean calculated.

\section{Statistical methods}

The accuracy of ultrasound measurements was quantified by comparing the B-scan measurements of the two-dimensional optic cup parameters with the corresponding measurements taken by the HRT. The relative error was defined as:

$$
\text { Relative error }=\frac{\text { ultrasound }}{\text { HRT value }} \times 100-100 \%
$$

The difference between corresponding readings taken on the two instruments was also calculated. The standard deviation (SD) and the mean of these differences was used to calculate the $95 \%$ limits of agreement between the two instruments (mean $\pm 2 \mathrm{SD}$ ), and Bland-Altman plots were drawn. This result indicates that, for $95 \%$ of observations, the difference between methods will fall within this range. The $95 \%$ confidence interval for the bias of echographic readings was also computed. ${ }^{4}$

To establish whether the size of the two-dimensional optic cup parameters influenced the accuracy of our measurements, a simple regression analysis between the HRT-ultrasound discrepancy and the subject mean was also performed. Where measurement errors were of normal distribution, reproducibility of the readings taken on each instrument was expressed in terms of the coefficient of repeatability. This represents the value below which the difference between two measurements will lie with probability 0.95 and is given by:

$$
\text { Coefficient of repeatability }=2 \times \sqrt{2 \times \mathrm{SD}^{2}}
$$

Test-retest variability was estimated by calculating the 95\% limits of agreement between initial and retest values. Reproducibility and test-retest variability were then compared for the two instruments.

Table 2. Accuracy of echographic measurements of two-dimensional optic cup parameters given by the correlation coefficient, relative error and

\begin{tabular}{|c|c|c|c|}
\hline & $\begin{array}{l}\text { Relative error } \\
(\text { mean } \pm S D)\end{array}$ & $\begin{array}{c}\text { Correlation coefficient } \\
\text { and } p \text { value }\end{array}$ & $\begin{array}{l}95 \% \text { limits of agreement } \\
\text { (mean difference } \pm 2 \text { SD) }\end{array}$ \\
\hline Maximum vertical optic cup diameter & $10.21 \pm 12.22 \%$ & $r=0.937 ; p<0.001$ & $95 \pm 210 \mu \mathrm{m}$ \\
\hline Maximum horizontal optic cup diameter & $-3.13 \pm 16.46 \%$ & $r=0.861 ; p<0.001$ & $19 \pm 156 \mu \mathrm{m}$ \\
\hline Maximum optic cup depth & $-11.43 \pm 24.18 \%$ & $r=0.774 ; p<0.001$ & $87 \pm 328 \mu \mathrm{m}$ \\
\hline
\end{tabular}
95\% limits of agreements between readings taken by ultrasound and scanning laser tomography 


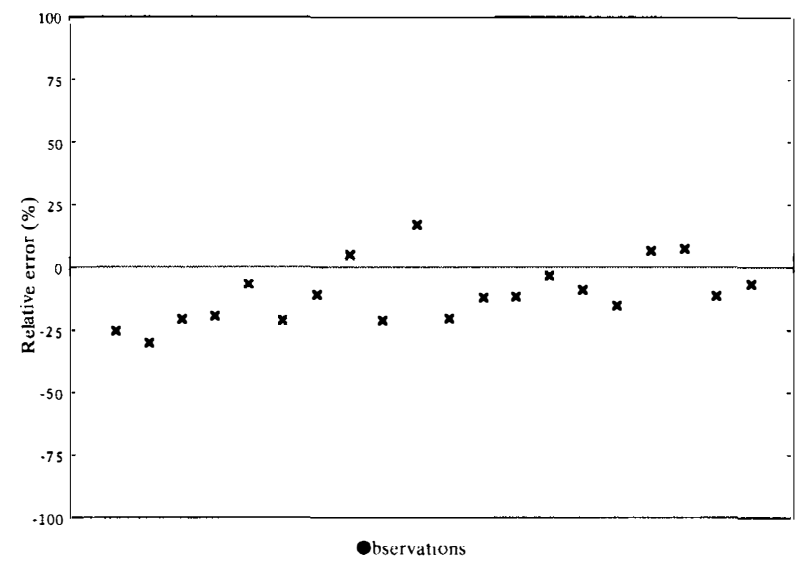

Fig. 3. Relative error of B-scan estimates of maximal vertical optic cup diameter with respect to HRT measurements.

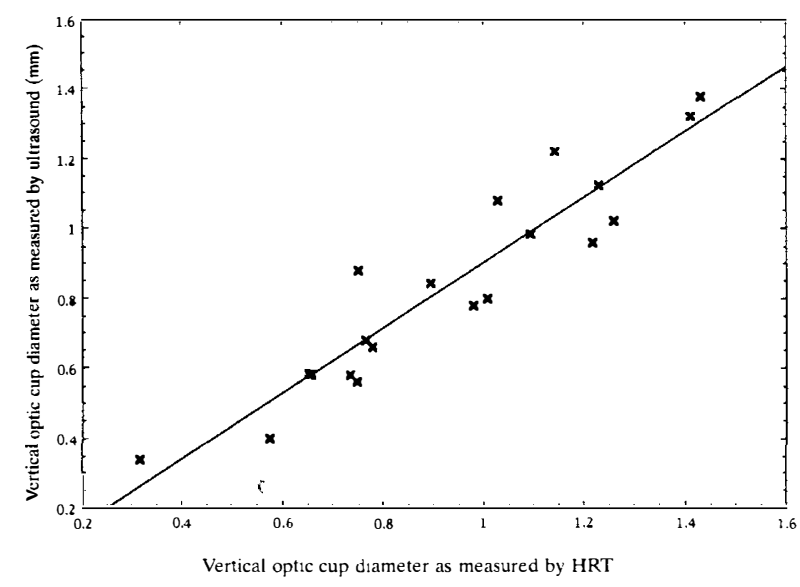

Fig. 4. Simple regression analysis of B-scan and HRT estimates of maximum vertical optic cup diameter.

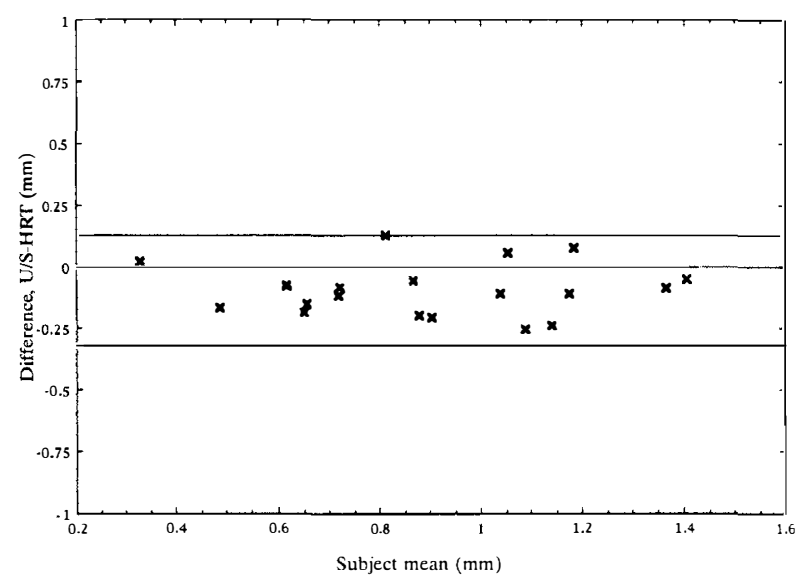

Fig. 5. Difference versus subject mean for vertical optic cup diameter measured by HRT and B-scan echography (Bland-Altman plot). The upper and lower $95 \%$ limits of agreement are indicated. U/S, ultrasound.

\section{Results}

We recruited 20 patients (16 glaucoma subjects, 4 ocular hypertension (OHT) subjects) into this study. Of these, 13 were male. The mean age of our sample $( \pm \mathrm{SD})$ was $62.25 \pm 13.7$ years $(\mathrm{OHT}, 69.5 \pm 10.6$ years; glaucoma,

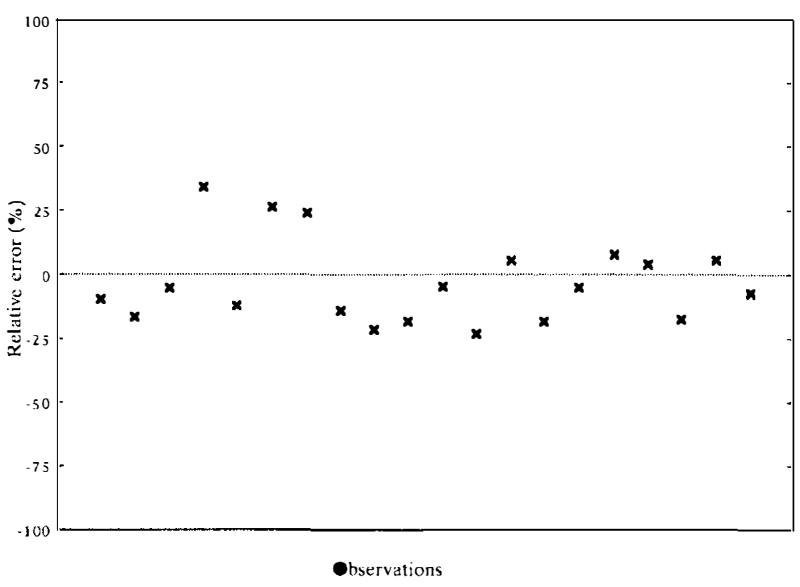

Fig. 6. Relative error of B-scan estimates of maximal horizontal optic cup diameter with respect to HRT measurements.

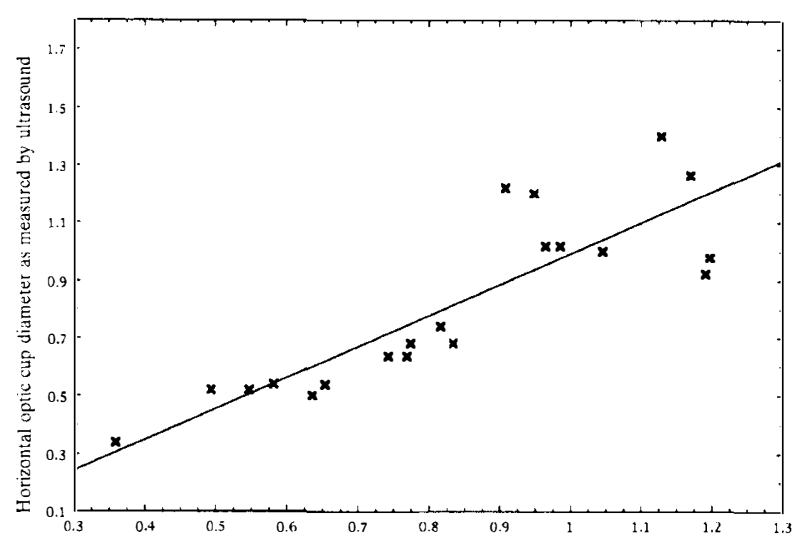

HRT measurements of horizontal optic cuip diameter $(\mathrm{mm})$

Fig. 7. Simple regression analysis of B-scan and HRT estimates of maximum horizontal optic cup diameter.

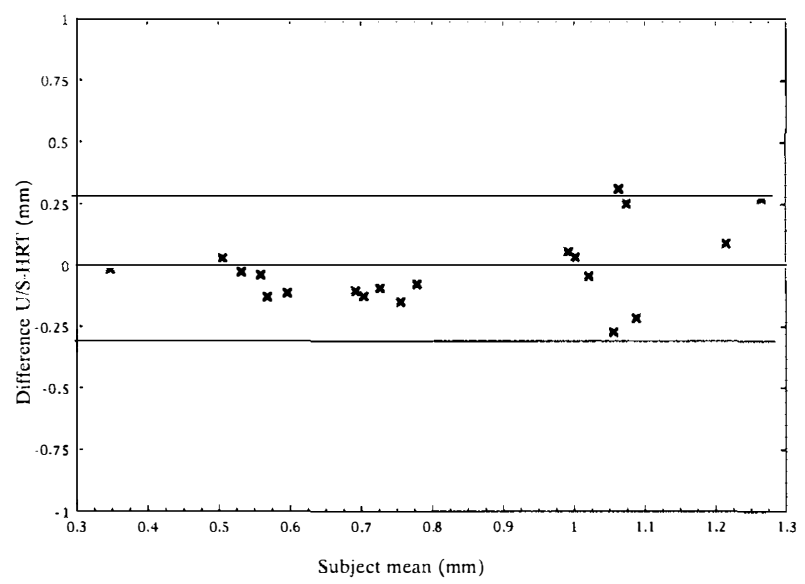

Fig. 8. Difference versus subject mean for horizontal optic cup diameter measured by HRT and B-scan echography (Bland-Altman plot). The upper and lower $95 \%$ limits of agreement are indicated. U/S, ultrasound.

$60.44 \pm 14.06$ years). The mean $\pm \mathrm{SD}$ refractive error [spherical equivalent] was $0.97 \pm 0.959$ dioptres (OHT, $0.062 \pm 0.427$ dioptres; glaucoma, $-0.143 \pm 1.073$ dioptres). Mean radius of corneal curvature ranged from 7.23 to $8.030 \mathrm{~mm}$ (mean $7.744 \mathrm{~mm}$ ). 


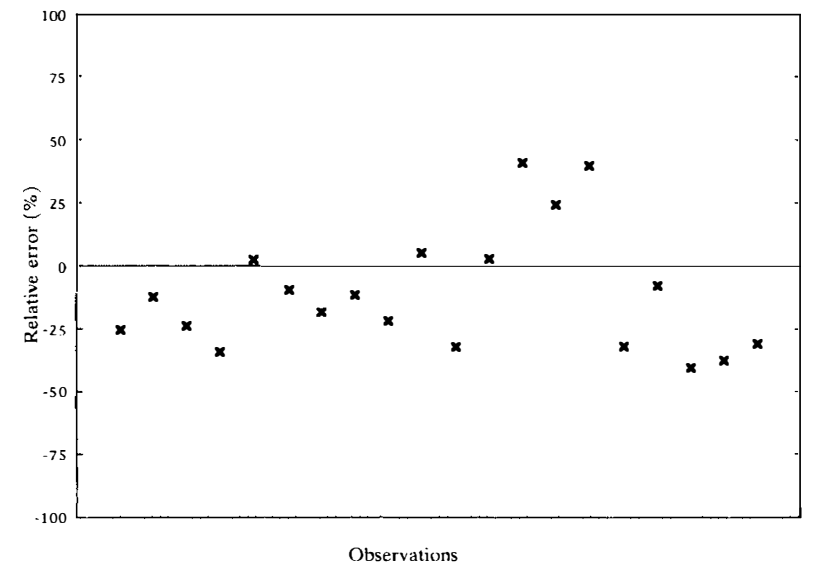

Fig. 9. Relative error of B-scan estimates of maximal optic cup depth with respect to HRT measurements.

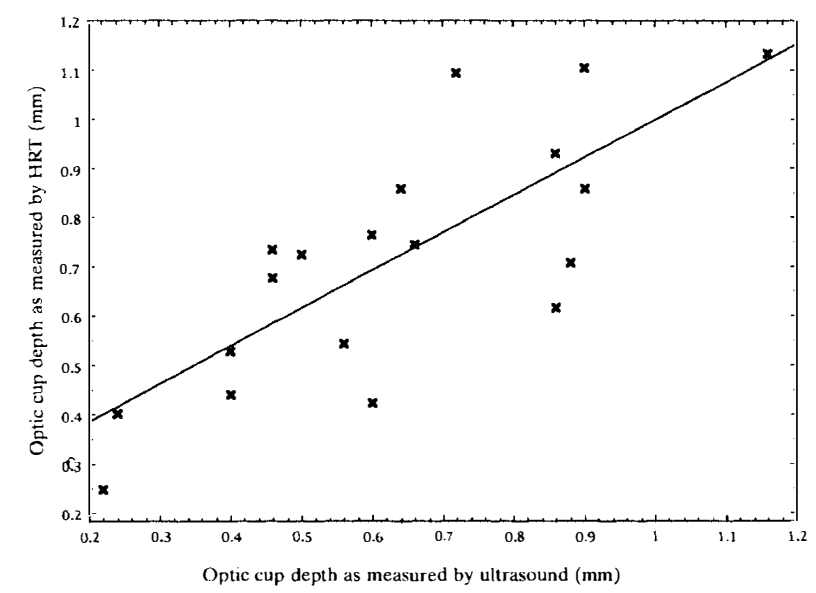

Fig. 10. Simple regression analysis of B-scan and HRT estimates of maximum optic cup depth.

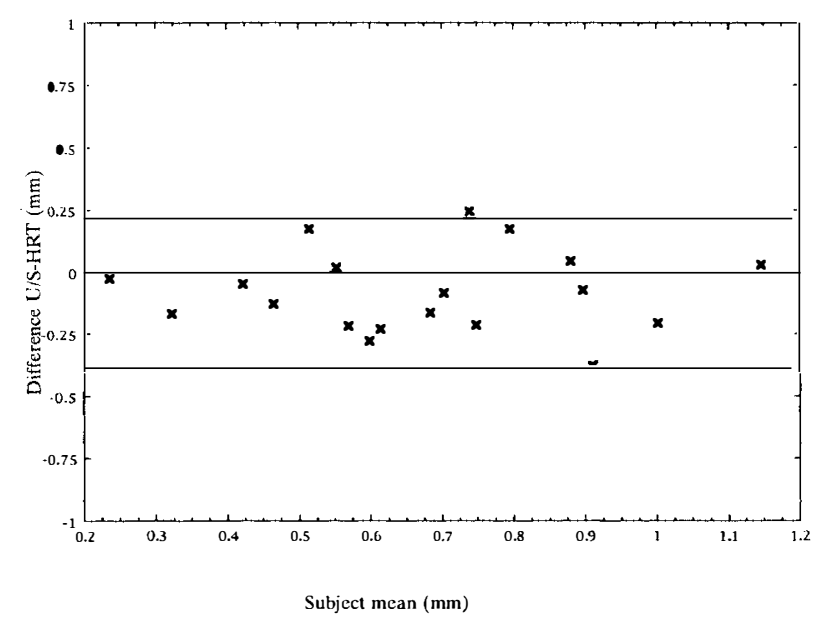

Fig. 11. Difference versus subject mean for optic cup depth measured by HRT and B-scan echography (Bland-Altman plot). The upper and lower $95 \%$ limits of agreement are indicated. U/S, ultrasound.

Measurements ranged from 316 to $1430 \mu \mathrm{m}$ (HRT) and from 340 to $1380 \mu \mathrm{m}$ (B-scan) for maximum vertical optic cup diameter (VOCD), from 357 to $1197 \mu \mathrm{m}$ (HRT) and from 340 to $1400 \mu \mathrm{m}$ (B-scan) for maximum horizontal optic cup diameter (HOCD), and from 250 to $1134 \mu \mathrm{m}$ (HRT) and from 220 to $1160 \mu \mathrm{m}$ (B-scan) for maximum cup depth (Table 1).
Accuracy of ultrasound readings (relative error) and agreement between readings taken on the two instruments (correlation coefficient and $95 \%$ limits of agreement) are given in Table 2 and displayed graphically in Figs. 3-11.

Discrepancies between HRT and ultrasound readings were independent of subject mean (VOCD [simple regression analysis]: $p=0.99$; HOCD: $p=0.08$; cup depth: $p=0.93$ ). The $95 \%$ confidence intervals for the bias of ultrasound readings were $95 \pm 48 \mu \mathrm{m},-19 \pm 72 \mu \mathrm{m}$ and $-87 \pm 328 \mu \mathrm{m}$ for VOCD, HOCD and cup depth measurements respectively.

Indices of reproducibility (coefficient of repeatability) and test-retest variability ( $95 \%$ limits of agreement between initial and retest values) are given in Table 1.

\section{Discussion}

Clinically, pathological optic disc cupping is distinguished from the physiological cup by loss of the symmetrical rim of normal-appearing disc tissue, increasingly evident laminar dots, ${ }^{5}$ the bayoneted appearance and nasal deviation of disc blood vessels, ${ }^{6}$ notching of the neuroretinal rim and interocular asymmetry of the cup/disc ratio. ${ }^{7}$

In the modern clinical setting, the optic disc is most commonly assessed using the 60,78 or 90 dioptre lens at the biomicroscope, and an estimate of the cup/disc ratio recorded in the medical notes. This method of monitoring optic disc cupping is unsatisfactory for several reasons. First, because the optic cup appears paler than surrounding disc tissue, there has been a tendency to define the cup in terms of colour change, which often does not reflect the cup as defined by configuration. ${ }^{8}$ Also, cup/disc ratio is an incomplete representation of the nature of the optic cup as it ignores depth and fails to provide a description of the shape of the cup. Furthermore, many factors influence the accuracy and reliability of slit-lamp estimates of disc morphometry including lens and individual ocular magnification factors, and inter- and intra-observer variability. ${ }^{9-11}$ Consequently, several methods designed to analyse optic disc morphometry without observer bias have been developed. These include planimetry, ${ }^{12,13}$ image analysis of stereoscopic photographs, ${ }^{14}$ optical coherence tomography, ${ }^{15}$ and confocal scanning laser tomography. ${ }^{116-19}$ Each technique has its own limitations, and all require expensive specialist equipment. Clear optic media and pupil dilatation are preferable if good image quality is to be obtained with any of these instruments.

The role of ultrasonography in the assessment of the optic cup has been previously investigated. ${ }^{20-22}$ In the first report, advanced cupping (cup/disc ratio $\geqslant 0.7$ ) was detectable as a concavity in the posterior pole. ${ }^{20}$ DarnleyFisch and co-workers ${ }^{21}$ showed that high-resolution contact B-scan could accurately categorise cups as small, medium or large. More recently Winder and $\mathrm{Atta}^{22}$ have shown a strong correlation between optical and ultrasonic cup diameters, and concluded that optic disc 
cups of $0.5 \mathrm{~mm}$ or larger could be defined by B-scan echography. However, the present study represents the first evaluation of optic cup measurements by a newgeneration, high-resolution, eye-dedicated B-scanner by comparing the results with HRT, the accuracy of which has been established. ${ }^{2,3}$

One obvious disadvantage of ultrasonic optic cup assessment is that the disc edge cannot be discerned by ultrasound, and therefore a cup/disc (C/D) ratio is not calculated. However, several studies investigating the correlation between structural optic disc measurements and functional impairment have failed to identify the C/D ratio as a reliable predictor of field loss. ${ }^{23,24}$ Discrim area and the third central moment of the frequency distribution of the depth values for the optic disc structures have, however, been shown to correlate strongly with visual field deficit. ${ }^{19,23,24}$

We investigated the reliability of two-dimensional optic cup data as measured by ultrasound, and assessed the agreement of these results with those of Heidelberg Retinal Tomography. We considered HRT values as 'actual values' in determining the relative error of ultrasound measurements. This assumption is based on the high degree of accuracy of laser tomography, estimated as $0.3-3.1 \%$ for cup diameter, $2 \%$ for cup area and $11.7 \%$ for cup depth measurements when verified against a model eye. ${ }^{2,3}$

We found echographic measurements of twodimensional optic cup data to be reliable, with indices of reproducibility and test-retest variability comparable to those of scanning laser tomography. The accuracy of these observations was also encouraging, reflected in the high degree of correlation between ultrasound and HRT readings for vertical and horizontal cup diameters and, to a lesser degree, for cup depth. The mean relative error for B-scan estimates of two-dimensional parameters was between $3 \%$ and $12 \%$. However, the $95 \%$ limits of agreement between the two methods (the range within which differences between corresponding readings taken on the two instruments will lie in $95 \%$ of cases) were not so small that the instruments can be used interchangeably. How small this difference has to be depends on the measurement and the use to which it is to be put. It is a clinical, not a statistical, decision. For purposes of glaucoma follow-up and the detection of subtle changes in optic nerve head morphometry over time, such as the annual loss of approximately $3 \%$ of neuroretinal rim area in glaucoma patients undergoing treatment, ${ }^{25}$ this degree of accuracy is clearly inadequate.

However, there remain many clinical situations where the two-dimensional measurements of the optic cup will contribute to patient care. First, a substantial increase in the B-scan estimate of a two-dimensional optic cup parameter over time (i.e. one which exceeds the limits of test-retest variability) can be considered as objective evidence of progression of cupping. Second, twodimensional optic cup parameters can now be reliably measured without the use of expensive specialist equipment and in the presence of media opacities. Although a pathological cup cannot be distinguished from a physiological cup on the basis of size alone, a small cup is clearly less likely to be pathological. Comparison with the fellow eye will also assist in the distinction. ${ }^{7}$ Patients with dense lens opacities awaiting cataract surgery and with an uncertain visual prognosis would benefit from such ultrasonic optic cup measurements, especially in those cases where extensive glaucomatous damage is suspected.

\section{References}

1. Rohrschneider K, Burk RO, Kruse F, Volcker HE. Reproducibility of the optic nerve head topography with a new laser tomographic scanning device. Ophthalmology 1994;101:1044-9.

2. Weinreb RN, Dreher AW. Reproducibility and accuracy of topographic measurements of the optic nerve head with the laser tomographic scanner. In: Nasemann JE, Burke ROW, editors. Scanning laser ophthalmoscopy and tomography. Munich: Quntessenz, 1990.

3. Dreher AW, Weinreb RN. Accuracy of topographic measurements in a model eye with the laser tomographic scanner. Invest Ophthalmol Vis Sci 1991;32:2992-6.

4. Bland M. An introduction to medical statistics, 2nd ed. New York: Oxford University Press, 1996.

5. Read R, Spaeth GL. Practical clinical appraisal of the optic disc in glaucoma: the natural history of glaucomatous cupping. Trans Am Acad Ophthalmol Otolaryngol 1974;78:255.

6. Scimeca HA. Optic disc changes in glaucoma. Int Ophthalmol Clin 1979;19:127-54.

7. Armaly MF. Cup/disc ratio in early open angle glaucoma. Doc Ophthalmol 1969;26:526.

8. Schwartz B. Cupping and pallor of the optic disc. Arch Ophthalmol 1973;89:272.

9. Spencer AF, Vernon SA. Optic disc height measurements with the Zeiss 4-mirror contact lens and 78 dioptre lens compared. Eye 1996;10:371-6.

10. Littman $\mathrm{H}$. The determination of the true size of objects in the background of the living eye. Klin Monatsbl Augenheilkd 1982;180:286-9.

11. Lichter PR. Variability of expert observers in evaluating the optic disc. Trans Am Ophthalmol Soc 1976;74:524-32.

12. Balazi AG, Drance SM, Schulzer M, Douglas GR. Neuroretinal rim area in suspected glaucoma and early chronic open-angle glaucoma. Arch Ophthalmol 1984;102:1011-4.

13. Bengtsson $B$. The variation and covariation of cup and disc diameters. Acta Ophthalmol (Copenh) 1976;54:804-18.

14. Takamoto T, Schwartz B. Reproducibility of photogrammetric optic disc cup measurements. Invest Ophthalmol Vis Sci 1985;26:814-7.

15. Hee MR, Izatt JA, Swanson EA, Huang D, Schuman JS, Lin $\mathrm{CP}$, et al. Optical coherence tomography of the human retina. Arch Ophthalmol 1995;113:325-32.

16. Chauhan BC, Le Blanc RP, McCormick TA, Rogers JB. Test-retest variability of topographic measurements with confocal scanning laser tomography in patients with glaucoma and control subjects. Am J Ophthalmol 1994;118:9-15.

17. Brigatti L, Weitzman M, Caprioli J. Regional test-retest variability of confocal scanning laser tomography. Am J Ophthalmol 1995;120:433-40.

18. Janknecht $P$, Funk J. Optic nerve head analyser and Heidelberg retina tomograph: accuracy and reproducibility of topographic measurements in a model eye and in a volunteer. Br J Ophthalmol 1994;78:760-8. 
19. Brigatti L, Caprioli J. Correlation of visual field with scanning confocal laser optic disc measurements in glaucoma. Arch Ophthalmol 1995;113:1191-4.

20. Cohen JS, Stone RD, Hetherington J, Bullock J. Glaucomatous optic cupping of the optic disc by ultrasonography. Am J Ophthalmol 1976;82:24-6.

21. Darnley-Fisch DA, Frazier Byrne S, Hughes JR, Parrish RK II, Feuer WJ. Contact B-scan echography in the assessment of optic nerve cupping. Am J Ophthalmol 1990;109:55-61.

22. Winder S, Atta HR. Ultrasonography of the optic disc cup in discs of various sizes. Eye 1996;10:732-6.
23. Funk J, Bornscheuer C, Grehn F. Neuroretinal rim area and visual field in glaucoma. Graefes Arch Clin Exp Ophthalmol 1988;226:431-4.

24. Jonas JB, Gusek GC, Naumann GO. Optic disk morphometry in chronic primary open-angle glaucoma. II. Correlation of the intrapapillary morphometric data to visual field indices. Graefes Arch Clin Exp Ophthalmol 1988;226:531-8.

25. Aireksinen PJ, Tuulonen A, Alanko HI. Rate and pattern of neuroretinal rim area decrease in ocular hypertension and glaucoma. Arch Ophthalmol 1992;110:20610. 